\title{
Combinatorial necklace splitting
}

\author{
Dömötör Pálvölgyi \\ Ecole Polytechnique Fédérale de Lausanne, Switzerland \\ dom@cs.elte.hu \\ Submitted: Jun 9, 2009; Accepted: Jun 24, 2009; Published: Jul 2, 2009 \\ Mathematics Subject Classification: 05A18,05D99,55M20

\begin{abstract}
We give a new, combinatorial proof for the necklace splitting problem for two thieves using only Tucker's lemma (a combinatorial version of the Borsuk-Ulam theorem). We show how this method can be applied to obtain a related recent result of Simonyi and even generalize it.
\end{abstract}

\section{$1 \quad$ Necklace Splitting}

This paper was inspired by the combinatorial proof of Matoušek [7] of the LovászKneser theorem [6]. He used Tucker's lemma [11] that was proved combinatorially by Freund and Todd [4]. A combinatorial proof for a generalization of Tucker's lemma can be found in a paper of Ziegler [12]. We start by stating a version of this lemma and then show how it can be used to give a simple proof for the necklace splitting theorem.

We denote the set $\{1, \ldots, n\}$ by $[n]$ and let $-S$ denote $\{-s: s \in S\}$, thus $-[n]=$ $\{-1, \ldots,-n\}$. For four subsets of $[n], A_{1}, B_{1}, A_{2}, B_{2}$ we say that the set-pair $\left(A_{1}, B_{1}\right)$ is smaller than the set-pair $\left(A_{2}, B_{2}\right)$ if $A_{1} \subset A_{2}$ and $B_{1} \subset B_{2}$ and at least one of the inclusions is strict. We denote this by $\left(A_{1}, B_{1}\right) \subset\left(A_{2}, B_{2}\right)$. A family of set-pairs is said to form a chain, if any two members of the family are comparable.

Lemma 1.1. (Octahedral Tucker's lemma, in [12] Lemma 4.1) If for any set-pair $A, B \subset$ $[n], A \cap B=\emptyset, A \cup B \neq \emptyset$ we have a $\lambda(A, B) \in \pm[n-1]$ color, such that $\lambda(A, B)=$ $-\lambda(B, A)$, then there are two set-pairs, $\left(A_{1}, B_{1}\right)$ and $\left(A_{2}, B_{2}\right)$, such that $\left(A_{1}, B_{1}\right) \subset$ $\left(A_{2}, B_{2}\right)$ and $\lambda\left(A_{1}, B_{1}\right)=-\lambda\left(A_{2}, B_{2}\right)$.

Two set-pairs for which $\left(A_{1}, B_{1}\right) \subset\left(A_{2}, B_{2}\right)$ and $\lambda\left(A_{1}, B_{1}\right)=-\lambda\left(A_{2}, B_{2}\right)$ are said to form an opposing inclusion. 
In the necklace splitting problem, we have an open necklace with $k$ types of beads, $a_{i}$ beads of the $i^{\text {th }}$ kind and $p$ thieves want to split it by using as few cuts as possible, such that each one of them gets $\left\lfloor a_{i} / p\right\rfloor$ or $\left\lceil a_{i} / p\right\rceil$ beads of the $i^{\text {th }}$ kind. They are allowed to cut the necklace between any two beads and then divide the parts among themselves arbitrarily. If the different types of beads are after each other, then it is easy to see that $(p-1) k$ cuts are necessary. That this number is always enough was proved for $p=2$ by Goldberg and West [5]. Later Alon and West [3] gave a simpler proof using the BorsukUlam theorem. This was generalized to arbitrary $p$, each kind having a multiple of $p$ number of beads by Alon [1] and the following version is the result of Alon, Moshkovitz and Safra [2].

Theorem 1.2. (Necklace Splitting Theorem) If $p$ thieves want to split a necklace with $k$ kinds of beads such that each of them gets $\left\lfloor a_{i} / p\right\rfloor$ or $\left\lceil a_{i} / p\right\rceil$ beads of the $i^{\text {th }} k i n d$, then they can do so using at most $(p-1) k$ cuts.

For another recent proof of this theorem see Meunier [8] (the proof is combinatorial but uses algebraic topological notions).

We conjecture that a generalization of this theorem might also be true. The generalization is that we can decide for each thief and each kind if the thief should get more or less beads of that kind. (So the statement is stronger in the case when $p$ does not divide the $a_{i}$ 's.)

Conjecture 1.3. (General Necklace Splitting Conjecture) If $p$ thieves want to split a necklace with $k$ kinds of beads such that the $j^{\text {th }}$ thief gets $a_{i}^{(j)}$ of the $i^{\text {th }}$ kind where $a_{i}^{(j)}=$ $\left\lfloor a_{i} / p\right\rfloor$ or $\left\lceil a_{i} / p\right\rceil$ and $\sum_{j} a_{i}^{(j)}=a_{i}$, then they can do so using at most $(p-1) k$ cuts.

We will give a direct, simple combinatorial proof from Tucker's lemma to this conjecture for the $p=2$ case in the next section. (In fact for the $p=2$ case, this conjecture follows easily from the previous theorem, this version is proved also in [9], but our proof method is quite different.) To demonstrate the power of our method, in Section 3 we reprove the result that appeared in a recent paper of Simonyi [10] following his idea but using our method. Finally in Section 4 we prove a generalization of it (Corollary 4.6) using some geometric observations about a property that we call stair-convexity. Theorem 4.8 at the end of that section is our strongest result.

\section{Proof of the classic necklace splitting theorem for two thieves}

Before the proof we need a definition, the core of the main idea.

Definition 2.1. For $A \cap B=\emptyset, A \cup B=[n]$ define $|m(A, B)|$ as the number of alternations between $A$ and $B$ when we are going from 1 to $n$ and the sign of $m(A, B)$ to be positive if $1 \in A$ and negative if $1 \in B$. For other set-pairs, define it as maxabs $\left\{m\left(A^{\prime}, B^{\prime}\right)\right.$ : $\left.(A, B) \subset\left(A^{\prime}, B^{\prime}\right), A^{\prime} \cap B^{\prime}=\emptyset, A^{\prime} \cup B^{\prime}=[n]\right\}$ where maxabs denotes the number with the largest absolute value. 
Eg. if $n=5, A=\{2\}, B=\emptyset$, then $|m(A, B)|=4$ because we can take $A^{\prime}=\{2,4\}$ and $B^{\prime}=\{1,3,5\}$. Note that the sign of $m(A, B)$ is well-defined because whether 1 belongs to $A^{\prime}$ or $B^{\prime}$ is determined by $A$ and $B$. Eg. $m(\{2\}, \emptyset)=-(n-1), m(\{3\},\{5,6\})=n-3$, while $m([n], \emptyset)=0$. Note that $|m(A, B)| \leq n-1$. Its connection to splitting necklaces is that if we associate the beads of the necklace with $[n]$ according to their order, then this number determines the maximum number of cuts with which we can provide a splitting of the necklace that lets the beads belonging to $A$ go to the first thief, those in $B$ to the second, while the ownership of the rest does not matter. Using this function, we can prove Conjecture 1.3 for $p=2$.

Theorem 2.2. (General Necklace Splitting for two thieves) If two thieves want to split a necklace with $k$ kinds of beads such that the $j^{\text {th }}$ thief gets $a_{i}^{(j)}$ of the $i^{\text {th }}$ kind where $a_{i}^{(j)}=\left\lfloor a_{i} / 2\right\rfloor$ or $\left\lceil a_{i} / 2\right\rceil$ and $a_{i}^{(1)}+a_{i}^{(2)}=a_{i}$, then they can do so using at most $k$ cuts.

Proof. Our goal is to give a $\lambda$ coloring fot the use Tucker's lemma. Denote the types of the beads by the numbers from 1 through $k$. Associate the beads of the necklace with $[n]$ according to their order. The first idea is that $\lambda(A, B)=+1$ if the number of the 1 beads in $A$ is more than $a_{1} / 2$, and it is -1 if the number of the 1 beads in $B$ is more than $a_{1} / 2$. If none of this happens, then $\lambda(A, B)=+2$ if the number of the 2 beads in $A$ is more than $a_{2} / 2$, and it is -2 if the number of the 2 beads in $B$ is more than $a_{2} / 2$, etc. This function is antipodal and it cannot have an opposing inclusion (since two disjoint sets cannot both have more than half of the beads of the same kind) but unfortunately it does not color all the set-pairs. So we have to extend it.

If $|m(A, B)| \geq k+1$, then let $\lambda(A, B)=m(A, B)$ and if $|m(A, B)| \leq k$, then let us keep our earlier definition, ie. $\lambda(A, B)=+1$ if the number of the 1 beads in $A$ is more than $a_{1} / 2$ etc. Note that we cannot have an opposing inclusion for a set-pair with $|\lambda(A, B)| \geq k+1$, because if we have an inclusion, then the extensions of the bigger setpair of the inclusion are also extensions for the smaller set-pair of the inclusion, thus the sign of the bigger must be the same as the sign of the smaller if their absolute values are the same. We have seen it earlier that no opposing inclusion can occur for $|\lambda(A, B)| \leq k$, thus using Tucker's lemma there must be an uncolored set-pair. For this set-pair we must have $|m(A, B)| \leq k$. Thus we have an assignment where for every $i$, nobody has more than $a_{i} / 2$ of the $i$ beads and no matter how we expand this assignment, the number of necessary cuts is always at most $k$. Therefore we can divide the rest of the beads in any way among the two thieves.

\section{Proof of Simonyi's result}

To demonstrate the power of our method, in this section we reprove the result that appeared in a recent paper of Simonyi [10].

Theorem 3.1. If two thieves want to split a necklace with $k$ kinds of beads, each kind having an even number, and we are given two disjoint subsets of $[k], D_{1}$ and $D_{2}$, not both 
empty, then they can split the necklace using at most $k-1$ cuts, such that the distribution is either completely even or the $j^{\text {th }}$ thief gets more beads of type $i$ if and only if $i \in D_{j}$.

He used a lemma, whose combinatorial equivalent is the following.

Lemma 3.2. (Octahedral Tucker-Bacon lemma) If for any set-pair $A, B \subset[n], A \cap B=$ $\emptyset, A \cup B \neq \emptyset$ we have an $\emptyset \neq \lambda(A, B) \subset \pm[n]$ color set, such that $\lambda(A, B)=-\lambda(B, A)$ and if $\left(A_{1}, B_{1}\right) \subset\left(A_{2}, B_{2}\right)$, then $\lambda\left(A_{1}, B_{1}\right) \cap-\lambda\left(A_{2}, B_{2}\right)=\emptyset^{1}$, then for any pair of disjoint subsets of $[n], I$ and $J$, not both empty, there is a chain of set-pairs, $\left\{\left(A_{h}, B_{h}\right)\right\}$ such that $\cup \lambda\left(A_{h}, B_{h}\right)=I \cup-J$.

This lemma easily follows from the continuous version and we will anyhow prove the same result without using this lemma, thus we omit its proof. Now we sketch how this lemma implies Simonyi's theorem using our method.

Proof. The main idea is the same as in our previous proof, we define a $\lambda$ function to use the Octahedral Tucker-Bacon lemma. If $m(A, B) \geq k$, then $\lambda(A, B)=\{m(A, B)+1\}$, if $m(A, B) \leq-k$, then $\lambda(A, B)=\{m(A, B)-1\}$. If $|m(A, B)|<k$, then $+i \in \lambda(A, B)$ if the number of the $i$ beads in $A$ is more than $a_{i} / 2$, and $-i \in \lambda(A, B)$ if the number of the $i$ beads in $B$ is more than $a_{i} / 2$. This way $\lambda(A, B)$ is indeed a subset of $\pm[n]$. Define $I=D_{1}$ and $J=D_{2}$ and apply the Octahedral Tucker-Bacon lemma (if something is uncolored, then there is a fair division with $k-1$ cuts and we are done). For the largest element of the chain that we get, we must have $\lambda(A, B)=I \cup-J$ because if someone already has the majority of the $i$ beads in a smaller set, he must also have it in a larger set. Any extension of this set has at most $k-1$ cuts thus it has an extension in which the majority of each type of bead from $I \cup J$ goes to the respective thief while the other types are divided evenly.

\section{Proof of a generalization}

To prove the generalization of Simonyi's result, first we need some geometric observations. Denote by $e_{i}$ the $i^{\text {th }}$ unit vector of the standard basis of $\mathbb{R}^{k}$.

Definition 4.1. We say that $S \subset \mathbb{Z}^{k}$ is stair-convex if between any two of its points $p, q$ there is a monotone path meaning that there exists a sequence, $\left\{p=r_{0}, r_{1}, \ldots, r_{h}=q\right\} \subset S$ and a suitable signing of the standard basis, $\epsilon_{i}= \pm e_{i}$ such that for any $j$ there is a $i$ such that $r_{j+1}-r_{j}=\epsilon_{i}$.

It is easy to see that the intersection of a stair-convex set with a line parallel to one of the axes is always an interval of integers. The intersection of a stair-convex set with an axis-parallel box is also a stair-convex set.

Definition 4.2. For a set $S \subset \mathbb{Z}^{k}$, let $S_{i}^{-}=\left\{z \in \mathbb{Z}^{k} \mid z \notin S, \exists t \in \mathbb{N} z+t e_{i} \in S\right\}$ and similarly $S_{i}^{+}=\left\{z \in \mathbb{Z}^{k} \mid z \notin S, \exists t \in \mathbb{N} z-t e_{i} \in S\right\}$.

\footnotetext{
${ }^{1}$ This is the analogue of not having an opposing inclusion for this multicolored version
} 
Claim 4.3. If $S$ is stair-convex, then $S_{i}^{-}$and $S_{i}^{+}$are disjoint. The set $S_{i}^{-} \cup S \cup S_{i}^{+}$is also stair-convex, and if $S$ is symmetric to the origin, then $S_{i}^{-}=-S_{i}^{+}$.

This claim implies that the sets $S^{[1]}=S_{1}^{-} \cup S \cup S_{1}^{+}, S^{[2]}=\left(S^{[1]}\right)_{2}^{-} \cup S^{[1]} \cup\left(S^{[1]}\right)_{2}^{+}, \ldots$, $S^{[k]}=\left(S^{[k-1]}\right)_{k}^{-} \cup S^{[k-1]} \cup\left(S^{[k-1]}\right)_{k}^{+}$are all stair-convex. It is clear that $S^{[k]}=\mathbb{Z}^{k}$ if $S \neq \emptyset$, moreover, already $S^{[k-1]}=\mathbb{Z}^{k}$ if $S$ intersects every hyperplane of the form $x_{k}=i$.

Claim 4.4. If $\ell$ is a line passing through the origin, then $L=\left\{z \in \mathbb{Z}^{k} \mid \exists p \in \ell\|p-z\|_{\infty}<\right.$ $1\}$ is a stair-convex set that is symmetric to the origin. Therefore $L^{[i]}$ is also a symmetric stair-convex set.

Now we are ready to prove the following theorem which gives a generalization of Simonyi's result (Theorem 3.1).

Theorem 4.5. Suppose two thieves want to split a necklace with $k$ kinds of beads, and we are given a sequence $\alpha_{1}, \ldots, \alpha_{k}$, not all zero. Then there is a $t \in \mathbb{R}$ such that they can split the necklace using at most $k-1$ cuts, such that the number of $i$ beads of the first thief minus the number of $i$ beads of the second thief is the closest integer to t $\alpha_{i}$ whose parity equals the parity of $a_{i}{ }^{2}$

Moreover, if none of the $\alpha_{i}$ 's is zero, then for $\ell=\{t \cdot \underline{\alpha}\}$ and $L=\left\{z \in \mathbb{Z}^{k} \mid \exists p \in\right.$ $\left.\ell\|p-z\|_{\infty}<1\right\}$ we can achieve that the point whose $i^{\text {th }}$ coordinate is the difference of the $i$ beads lays in $L$.

Corollary 4.6. Suppose two thieves want to split a necklace with $k$ kinds of beads, each kind having an even number, and we are given two disjoint subsets of $[k], D_{1}$ and $D_{2}$, not both empty. Then there is a $d \in \mathbb{N}$, such that they can split the necklace using at most $k-1$ cuts, such that the number of $i$ beads of the first thief is $a_{i} / 2+d$ for $i \in D_{1}, a_{i} / 2-d$ for $i \in D_{2}$ and $a_{i} / 2$ for $i \notin D_{1} \cup D_{2}$.

Proof. (of the corollary.) Choose $\alpha_{i}=1$ for $i \in D_{1}, \alpha_{i}=-1$ for $i \in D_{2}$ and $\alpha_{i}=1 / 2 n$ for $i \notin D_{1} \cup D_{2}$. The theorem gives us a $z \in \mathbb{Z}^{k}$ and a $t \in \mathbb{R}$ such that $\|t \cdot \underline{\alpha}-z\|_{\infty}<1$. Since every $a_{i}$ is even, the differences in the number of beads, $z_{i}$, will be also even. For $i, j \in D_{1}$ we have $\left|z_{i}-z_{j}\right| \leq\left|z_{i}-t\right|+\left|t-z_{j}\right|<1+1$, thus $z_{i}=z_{j}$. For $i \in D_{1}, j \in D_{2}$ we have $\left|z_{i}+z_{j}\right| \leq\left|z_{i}-t\right|+\left|t+z_{j}\right|<1+1$, thus $z_{i}=-z_{j}$. Since $z_{i} \leq n$, we also know that $t<n+1$. For $i \notin D_{1} \cup D_{2}, 1>\left|z_{i}-t / 2 n\right|>\left|z_{i}\right|-|(n+1) / 2 n|$ and the parity implies $z_{i}=0$. This proves the statement (after a possible swap of the rôles of the thieves.)

Proof. (of the theorem.) The proof will be very similar to the proof of the normal necklace splitting theorem. Let $\ell$ be $\{t \cdot \underline{\alpha}\}$ and $L=\left\{z \in \mathbb{Z}^{k} \mid \exists p \in \ell\|p-z\|_{\infty}<1\right\}$. Then $L, L_{1}^{-}, L_{1}^{+}, \ldots,\left(L^{[k-2]}\right)_{k-1}^{-},\left(L^{[k-2]}\right)_{k-1}^{+}$give a partitioning of the space (unless $\alpha_{k}=0$ but we can suppose without loss of generality that this is not the case). For simplicity, define $H_{i}=\left(L^{[k-i-1]}\right)_{k-i}^{+}$and $-H_{i}=\left(L^{[k-i-1]}\right)_{k-i}^{-}$as the only property that we will use is that $\mathbb{Z}^{k} \backslash \cup_{j<i}\left(H_{j} \cup-H_{j}\right)$ is stair-convex. (And define $L^{[0]}=L$, so that $H_{k-1}=L_{1}^{+}$.)

\footnotetext{
${ }^{2}$ If there are two such integers, then one of them.
} 
For any set-pair $(A, B)$ we define a mapping $\varphi$ to $\mathbb{Z}^{k}$ where $\varphi_{i}$ equals the number of $i$ beads in $A$ minus the number of $i$ beads in $B$. We say that $(A, B)$ avoids $S$ if $\varphi(A, B) \notin S$. Now we can define $\lambda$.

If $|m(A, B)| \geq k$, then $\lambda(A, B)=m(A, B)$. If $|m(A, B)|<k$, then we define $\lambda$ as follows. If the $\varphi$ of every extension of $(A, B)$ is in $H_{1}$, then $\lambda(A, B)=+1$ and if every extension is in $-H_{1}$, then $\lambda(A, B)=-1$. If the $\varphi$ of every extension of $(A, B)$ that avoids $H_{1} \cup-H_{1}$, is in $H_{2}$, then $\lambda(A, B)=+2$ and if every such extension is in $-H_{2}$, then $\lambda(A, B)=-2$. Generally, we define $\lambda(A, B)= \pm i$ if the $\varphi$ of every extension of $(A, B)$ that avoids $\cup_{j<i}\left(H_{j} \cup-H_{j}\right)$, is in $\pm H_{i}$.

Claim 4.7. If $|m(A, B)|<k$ and $|\lambda(A, B)| \not \leq i$, then it has an extension that avoids $\cup_{j \leq i}\left(H_{j} \cup-H_{j}\right)$.

Proof. By induction, we can suppose that $(A, B)$ has an extension that avoids $\cup_{j<i}\left(H_{j} \cup\right.$ $\left.-H_{j}\right) \cup H_{i}$ and another that avoids $\cup_{j<i}\left(H_{j} \cup-H_{j}\right) \cup-H_{i}$. Since $\mathbb{Z}^{k} \backslash \cup_{j<i}\left(H_{j} \cup-H_{j}\right)$ is stairconvex, between any two of its points there is a monotone path. The $\varphi^{\prime}$ s of the possible extensions of $(A, B)$ form a box in $\mathbb{Z}^{k}$, if we intersect this box with $\mathbb{Z}^{k} \backslash \cup_{j<i}\left(H_{j} \cup-H_{j}\right)$, we get a stair-convex set. When going from the extension avoiding $H_{i}$ to the one avoiding $-H_{i}$ on the monotone path inside this stair-convex set, somewhere on the way we must avoid both $H_{i}$ and $-H_{i}$ because of Claim 4.3.

It is easy to see that $\lambda$ satisfies the conditions of Tucker's lemma and that we cannot have an opposing inclusion, thus it is somewhere undefined. No matter how we extend this assignment, the number of necessary cuts is always at most $k-1$ and it has an extension that avoids all the $H_{i}$ 's because of the previous claim. We can divide the rest of the beads such that we stay in the closure of $L$ and (moreover) if $\alpha_{i} \neq 0$ for all $i$, then we can even stay in $L$. We are done.

Of course the same proof works for a much larger class of functions, instead of $L$, we could have omitted the integers close to $y=x^{3}$ or a $d$-dimensional subspace. In fact, we have proved the following theorem.

Theorem 4.8. If a stair-convex set $S \subset \mathbb{Z}^{k}$, that is symmetric to the origin, intersects every integer affine subspace that is perpendicular to $e_{j_{1}}, \ldots e_{j_{d}}$ for some $j_{1}, \ldots, j_{d}$, then we can split any necklace among two thieves with $k-d$ cuts such that there is an $s \in S$ such that the number of $i$ beads of the first thief minus the number of $i$ beads of the second thief is the closest integer to the $i^{\text {th }}$ coordinate of $s$ whose parity equals the parity of $a_{i}{ }^{3}$

Moreover, if $\forall i$ and $s \in S$ either $s+e_{i} \in S$ or $s-e_{i} \in S$, then we can achieve that the point whose $i^{\text {th }}$ coordinate is the difference of the $i$ beads lays in $S$.

\section{$5 \quad$ Remarks and acknowledgment}

I am thankful to Gábor Simonyi for his very useful advice and finding several mistakes in the earlier versions of this paper. I am also thankful to Zoltán Király for early discussions

\footnotetext{
${ }^{3}$ If there are two such integers, then one of them.
} 
on the subjest and to Gábor Tardos for disproving one of my earlier conjectures, that would have stated that $(p-1) k$ cuts are enough even if we demand that the thieves follow each other in cyclic order, eg. for $p=3, k=2$ the parts must belong to the 1., 2., 3., 1.,2. thief (where any part can be empty too). This suggests that the combinatorial proof might not work for bigger $p$ 's, not even if we use $Z_{p}$-Tucker's lemma or some other analogue. However, we cannot resist to state another conjecture that is stronger than the General Necklace Splitting Conjecture.

Conjecture 5.1. (Strong Necklace Splitting Conjecture) If p thieves want to split a necklace with $k$ kinds of beads such that the $j^{\text {th }}$ thief gets $a_{i}^{(j)}$ of the $i^{\text {th }}$ kind where $a_{i}^{(j)} \geq\left\lfloor a_{i} / p\right\rfloor$ and $\sum_{j} a_{i}^{(j)}=a_{i}$, then they can do so using at most $(p-1) k$ cuts.

Those who are familiar with the proof of the Necklace Splitting Theorem should note that even if one can prove this conjecture for prime $p$ 's, it does not imply automatically that it also holds for composite $p$ 's as in that proof.

Are these conjectures true for at least $p=3$ ?

Is there a simple characterization for the sets $S$ for which it is true that we can split any necklace among two thieves with $k-d$ cuts such that there is an $s \in S$ such that the number of $i$ beads of the first thief minus the number of $i$ beads of the second thief is the $i^{\text {th }}$ coordinate of $s$ ?

\section{References}

[1] N. Alon, Splitting necklaces, Advances in Mathematics 63 (1987), 247-253.

[2] N. Alon, D. Moshkovitz and M. Safra, Algorithmic construction of sets for k-restrictions, ACM Transactions on Algorithms 2 (2006), 153-177.

[3] N. Alon and D. B. West, The Borsuk-Ulam Theorem and bisection of necklaces, Proc. Amer. Math. Soc. 98 (1986), 623-628.

[4] R. M. Freund, M. J. Todd: A Constructive Proof of Tucker's Combinatorial Lemma. J. Comb. Theory, Ser. A 30(3): 321-325 (1981).

[5] C. H Goldberg and D. B. West, Bisection of circle colorings, SIAM J. Algebraic Discrete Methods 6 (1985), 93-106.

[6] L. Lovász, Kneser's Conjecture, Chromatic Numbers and Homotopy, J. Comb. Th. A 25, 319-324, 1978.

[7] J. Matoušek, A combinatorial proof of Kneser's conjecture, Combinatorica 24 (1) (2004), 163-170.

[8] F. Meunier, Polytopal complexes: maps, chain complexes and... necklaces, arXiv:0806.1488v1

[9] F. Meunier, Discrete Splittings of the Necklace, Math. of Operation Research, 33, 678-688, 2008. 
[10] G. Simonyi, Necklace bisection with one cut less than needed, Electron. J. Combin., 15 (2008), no. 1, Note 16.

[11] A.W. Tucker, Some topological properties of disk and sphere, Proceedings of the First Canadian Mathematical Congress, Montreal, 1945, University of Toronto Press, Toronto, 1946, pp. 285-309.

[12] G. M Ziegler, Generalized Kneser coloring theorems with combinatorial proofs, Inventiones Math. 147 (2002), 671-691. 